\title{
EXPERIMENTAL MODELS OF PULMONARY FIBROSIS AND THEIR TRANSLATIONAL POTENTIAL
}

\author{
Adamcakova Jana ${ }^{1}$, Palova Romana ${ }^{1}$, Mokra Daniela ${ }^{1}$ \\ ${ }^{1}$ Department of Physiology, Jessenius Faculty of Medicine in Martin, Comenius University \\ in Bratislava, Martin, Slovakia
}

\begin{abstract}
A b s tract
Pulmonary fibrosis, represented mainly by idiopathic pulmonary fibrosis, develops chronic and progressive changes in lung parenchyma with high mortality and limited therapeutic options. The aim of this review was to summarize the most common experimental models used in the research of pulmonary fibrosis. Lung damage associated with development of pulmonary fibrosis can be caused by irradiation or by instillation of bleomycin, fluorescein isothiocyanate (FITC), silicon dioxide (silica), asbestos, etc. This article reviews the characteristics of the most frequently used animal models of fibrosis, including the limitations of their use. Although none of the used animal models resembles completely the changes in human pulmonary fibrosis, similarities between them allow preclinical testing of novel treatment approaches or their combinations in the laboratory conditions before their use in the clinical practice.
\end{abstract}

Key words: animal model, idiopathic pulmonary fibrosis, bleomycin, fluorescein isothiocyanate (FITC), asbestos

\section{INTRODUCTION}

\section{Pulmonary fibrosis}

Pulmonary fibrosis, represented mainly by idiopathic pulmonary fibrosis (IPF), develops chronic and progressive changes in lung parenchyma with high mortality and limited therapeutic options. IPF as a representant of pulmonary fibrosis is a chronic pulmonary disease with a poor prognosis that can be often worse than many types of cancer. This disease occurs predominantly in adult individuals, to a greater extent in males, and is solely limited to lungs. IPF has been associated with the histopathological and/or radiological image of usual interstitial pneumonia (UIP) (1). It is anticipated that the initial stages of the disease include in particular the chronic inflammation; therefore, corticosteroids and other drugs with anti-inflammatory and immunosuppressive effects are used at this stage of the disease. Lung tissue fibrosis occurs in the later stage of the disease where antifibrotic treatment (e.g. pirfenidone or nintedanib) is recommended, but these drugs only prevent the disease from deteriorating. Additional non-pharmacological approaches such as long-term oxygen therapy, pulmonary rehabilitation, or lung transplantation can be used (2), as well.

\section{Experimental models of pulmonary fibrosis}

Animal models play an important role in the exploration of diseases because they allow understanding of the pathophysiological mechanisms of their formation in in vivo conditions. An ideal animal model should be the most imitating human illness, be highly reproducible and consistent, easy to execute, widely accessible, and not too expensive. The general benefits of animal models are related to the ability to reproduce complex genetic, biochemical, environmental, and phenotypic interactions. Creating animal models for chronic diseases is particularly challenging. Even more complicated is a situation with idiopathic

Corresponding author: Jana Adamcakova; e-mail: adamcakova5@uniba.sk

(C) 2019 Adamcakova J. et al.

This work is licensed under the Creative Commons Attribution-NonCommercial-NoDerivs 4.0 License

(https://creativecommons.org/licenses/by-nc-nd/4.0/) 
pulmonary fibrosis (IPF) because the etiopathology is unclear and no trigger is known to induce all known IPF features on animals.

Over the years, however, various pre-clinical models of pulmonary fibrosis have been developed, which have been evoked using different animal species (mice, rats, dogs, guinea pigs, dogs, sheep). The most common are mice and rats due to the ease of handling, availability of reagents, and well-characterized immune systems $(1,3)$. However, use of mice and rats is limited by the differences of their lungs to those of human, small size, as well as by their reduced sensitivity to cough $(1,3)$. For instance, use of larger animals could enable to use a fibre-optic bronchoscope to deliver fibrogenic factor (e.g. bleomycin) topically to induce a more severe fibrosis in selected segments, which is more akin to pulmonary fibrosis in patients, while the whole-lung exposure to fibrogenic agent to smaller animals can be associated with more severe tissue damage and higher mortality of animals (3). In addition, use of larger animals enables to use additional investigational methods including high-resolution CT imaging or repetitive measurement of lung functions including gas exchange or to examine the histopathological changes and collagen content from the lung biopsies (3). Thereby, the progression of pulmonary fibrosis can be assessed more reliably on a smaller number of larger animals than on numerous small animals (3).

Pulmonary fibrosis can be caused by different fibrotic insults, routes of administration, and dosing regimes (single or repeated administration). Most of these models mimic some

Table 1. Advantages and disadvantages of selected animal models of pulmonary fibrosis

\begin{tabular}{|c|c|c|}
\hline Model & Advantages & Disadvantages \\
\hline Bleomycin & $\begin{array}{l}\text { Best characterized } \\
\text { Can be delivered intratracheally, } \\
\text { intravenously, intraperitoneally, } \\
\text { subcutaneously, or intranasally } \\
\text { Clinically relevant model } \\
\text { Time frame for development } \\
\text { fibrosis 14-28 days }\end{array}$ & $\begin{array}{l}\text { Fibrosis in mice is reported to be self- } \\
\text { limiting after } 28 \text { days (in the intratra- } \\
\text { cheal model) }\end{array}$ \\
\hline $\begin{array}{l}\text { Fluorescein } \\
\text { isothiocyanate (FITC) }\end{array}$ & $\begin{array}{l}\text { Ability to visualize areas of lung } \\
\text { injury by characteristic green } \\
\text { fluorescence } \\
\text { The time frame for development } \\
\text { fibrosis } 14-28 \text { days } \\
\text { Fibrotic response persists for at } \\
\text { least } 6 \text { months } \\
\text { Relatively reproducible }\end{array}$ & $\begin{array}{l}\text { The response can vary depending on } \\
\text { the lot of FITC } \\
\text { The solution must be made fresh each } \\
\text { day and vortexed before each injection } \\
\text { Model is not clinically relevant } \\
\text { Missing representative UIP lesions }\end{array}$ \\
\hline $\begin{array}{l}\text { Radiation-induced } \\
\text { fibrosis }\end{array}$ & Clinically relevant model & $\begin{array}{l}\text { More than } 30 \text { weeks to develop fibrosis } \\
\text { Expensive per diem costs }\end{array}$ \\
\hline $\begin{array}{l}\text { Silica } \\
\text { (Silicon dioxide) }\end{array}$ & $\begin{array}{l}\text { Can be delivered oropharyngeal- } \\
\text { ly, by inhalation, or by intratra- } \\
\text { cheal aspiration } \\
\text { Persistent fibrotic stimulus }\end{array}$ & $\begin{array}{l}12-16 \text { weeks to fully developed fibrosis } \\
\text { BALB/c mice are resistant } \\
\text { Special instrumentation is needed if } \\
\text { delivered via aerosol } \\
\text { Model is not reproducible } \\
\text { Missing representative UIP lesions }\end{array}$ \\
\hline Asbestos & $\begin{array}{l}\text { Can be delivered by inhalation or } \\
\text { intratracheally }\end{array}$ & $\begin{array}{l}\text { More than } 1 \text { month to develop fibrosis } \\
\text { in inhalation model } \\
\text { Unequal distribution in the lungs after } \\
\text { simple intratracheal administration }\end{array}$ \\
\hline
\end{tabular}

(Adapted from Ref. 1, 3, 4). 
but never all of the characteristics of human IPF, especially the progressive and irreversible nature of this condition. Commonly used methods of induction of pulmonary fibrosis include lung damage by irradiation or instillation of bleomycin, fluorescein isothiocyanate (FITC), silicon dioxide (silica), or asbestos, with bleomycin being the most common inducer of experimental pulmonary fibrosis in animals $(1,3,4)$ (Table 1).

\section{Bleomycin model}

Bleomycin model is the most frequently used model of pulmonary fibrosis; therefore, we decided to discuss this model in a more detailed manner. Bleomycin is a chemotherapeutic antibiotic with anticancer activity that was discovered by Umezawa in 1966. Its structure has been known since 1978 (5) and the total synthesis was performed in 1982 (6). The use of bleomycin to induce pulmonary fibrosis in animal models is based on the fact that fibrosis is one of the major adverse effects of bleomycin in the treatment of human cancers. Bleomycin plays an important role in the treatment of lymphoma, squamous cell carcinomas, germ cell tumours, and malignant pleural effusion which it is injected intrapleurally. It is assumed that bleomycin causes breaks in one or both strands of DNA in the cancer cells, resulting in disruption of the cell cycle. This is probably due to chelation of metal ions and reaction of pseudo-enzyme with oxygen, resulting in the production of DNA-cleaving superoxides and hydroxide free radicals $(7,8)$. Overproduction of reactive oxygen species can lead to an inflammatory response causing pulmonary toxicity, fibroblast activation, and subsequent fibrosis (9). Bleomycin hydrolase, a bleomycin-inactivating enzyme, critically impacts the effects of this drug on various tissues. Lungs maintain low levels of this enzyme; therefore, they are susceptible to bleomycin-induced tissue damage (10). Until now, mechanisms associated with the development of pulmonary fibrosis have not been fully known. Several studies have analysed various potential biological and molecular factors such as transforming growth factor beta-1 (TGF-1), tumour necrosis factor alpha (TNF- $\alpha$ ), extracellular matrix components, chaperones, interleukins, and chemokines (6). In addition, bleomycin may cause generalized endothelial damage and imbalance in the fibrinolytic system (11).

Bleomycin has been shown to induce lung damage in various animal species - mice, hamsters, rats, rabbits, guinea pigs, dogs, and primates (12-14). Although the effects of bleomycin have been investigated in many animal models, the results from these studies are highly inhomogeneous because bleomycin is administered in different doses and different routes. In intravenous delivery of bleomycin the initial lesions appear to the pulmonary endothelium and the epithelial damage including necrosis of type I cells, metaplasia of type II cells and deposition of collagen in the lungs occurs subsequently. However, fibrotic changes need 4-12 weeks to develop, while in some animals they can be absent (1). Nowadays, bleomycin at a dose of $1.25-4 \mathrm{U} / \mathrm{kg}$ is administered in general anaesthesia mainly via the intratracheal route - by direct transtracheal injection or by naso- or oro-tracheal instillation. Transtracheal injection requires a midline incision in the neck area which is sutured immediately after the injection of bleomycin. Transoral instillation is a nonsurgical alternative when bleomycin is given into the trachea via a transoral route during positioning of the animal on the angled surgery board $(15,16)$. After bleomycin instillation the animals should be carefully monitored, with daily measurement of body weight and observation of their appearance, behaviour, and physiological measures. Of course, a special attendance should be paid to animals after surgical intervention. However, because the transoral method of delivery is less injurious for animals with lower mortality and post-delivery complications while having comparable distribution of bleomycin throughout the lungs, this delivery approach should be preferred not only for bleomycin model but also for other models where lung fibrosis is induced by intratracheal delivery of fibrogenic agent.

Bleomycin causes inflammatory and fibrotic reactions within a short time interval. In case of intratracheal administration, its action is even faster. The initial increase in proin- 
flammatory cytokines (interleukin (IL)-1, TNF- $\alpha$, IL-6, interferon- $\gamma$ ) is followed by an increased expression of profibrotic markers (TGF- $\beta 1$, fibronectin, procollagen- 1 ) with a peak around 14 days after bleomycin administration. Inflammatory changes are gradually replaced by fibrotic changes around the 9th day after bleomycin administration (9). In past the bleomycin model helped to clarify the role of cytokines, growth factors and signaling pathways that are likely to be involved in pulmonary fibrosis (17). In addition, observations of changes in the bleomycin model helped to determine that TGF- $\beta$ is one of the key factors in the development of pulmonary fibrosis. Subsequently, this model was used in the preclinical testing of nintedanib and pirfenidone (18-21) and thereby contributed to their introducing in the clinical practice. However, the bleomycin model is essential for development and testing of variety of potentially beneficial treatments inhibiting TGF- $\beta$ signalling (22-25), as well.

Advantages and disadvantages of the model: Bleomycin model is well-characterized, quite simple and rapid to develop, is reliable and reproducible, and, thus, it fulfills all the important criteria which are expected out of a good animal model. However, the development of fibrosis in mice is at least partially reversible beyond 28 days, irrespective of the type of intervention $(26,27)$. The problem of spontaneous resolution could be theoretically solved by repetitive administration of lower bleomycin doses. However, longer persistence of fibrosis after this procedure was associated with significant increase in mortality of animals (28). Since the bleomycin model lacks an aspect of the slow and irreversible development typical of IPF in patients (29), this fact must be taken into account, e.g. in pre-clinical drug testing.

\section{FITC model}

Fluorescein isothiocyanate (FITC) is another substance used to evoke pulmonary fibrosis. When the FITC is administered directly into the airways, fluorescein acts as a hapten and attaches to other lung proteins and acts as a prolonged form. The fibrotic response to FITC develops over 2 to 4 weeks and can last up to 24 weeks (30). The main advantage of FITC molecule is its fluorescence property that can identify the immunofluorescence areas of deposition and surrounding fibrosis in a given tissue. This model was described for the first time by Roberts et al. in 1995 (31). Intratracheal administration of FITC to BALB/c mice caused a significant infiltration of mononuclear cells and neutrophils into the lung interstitium, primarily focused around respiratory bronchioles, and a generation of oedema and hyperplasia of alveolar epithelial cells. A significant increase in proteins concentration in the bronchoalveolar lavage fluid within the 1st week confirmed a generation of an acute lung injury (32). FITC-induced model is dependent on Th2-lymfocytes producing profibrotic IL-13 in lungs.

Advantages and disadvantages of the model: FITC causes fibrotic changes in time relations similar to bleomycin and an additional advantage is its easy visualization by green fluorescence. However, there are several disadvantages: the solution of FITC must be prepared immediately before each administration, effectiveness of FITC can vary depending on the used batch and on the size of particles (30), and the fibrotic response also highly varies with an absence of several histopathological features including fibroblast foci (33).

\section{Radiation-induced fibrosis model}

Pulmonary fibrosis may be also induced by some non-chemical approaches in vivo, e.g. exposure to chest radiation. Radiation-induced fibrosis is dependent on the strain used in mice, with C57Bl/6 being the most sensitive (34). It is a relatively slow procedure that leads to the formation of fibrosis within 24 weeks (35). Irradiation causes a direct cell death of pneumocytes types I and II by DNA damage, followed by migration of macrophages into the damaged areas. Activated mononuclear cells then produce proinflammatory and profibrotic cytokines including TNF- $\alpha$ and TGF- $\beta$ that are involved in the development of fibrosis. The 
rate of fibrosis is dependent on the dose of radiation, e.g. a dose of 10 to 20 Gy was used in mice (36). Only one strong radiation effect is sufficient to induce the fibrotic process, but it may take up to 6 months for fibrosis to develop (35).

Advantages and disadvantages of the model: This model is advantageous due to its ability to induce persistent fibrosis-like changes in lungs. However, the formation of fibrosis in this way takes a relatively long time and the costs of irradiation (including technical equipment for irradiation) can be high. This model also lacks some of the histopathological features observed in IPF patients such as complex fibroblast deposits (37).

\section{Silica model}

Inhalation of silicate particles also leads to pulmonary fibrosis. The silica micro- or nanoparticles at a dose of 6-50 $\mathrm{mg}$ in $1 \mathrm{ml}$ of saline per kg body weight can be experimentally administered via oropharyngeal, inhalation, or intratracheal aspiration (38). In terms of silica sensitivity, the murine $\mathrm{C} 3 \mathrm{H} / \mathrm{HeN}$ and $\mathrm{C} 57 \mathrm{Bl} / 6$ mice are the most suitable for this type of experiment. Silica induces development of fibrotic nodules that occur at the earliest 4 weeks after the administration. Although this model can be used to monitor fibrotic mediators, it does not uniquely replicate the nature of IPF. The extent of fibrosis is dependent on the kinetics of the supplied silica, which is highly heterogeneous, and also depends on the mode of administration, the dosage regimens, and the silica particle formulations. Creating intratracheal models is faster (fibrosis develops within 14-28 days) and cost-effective, while the aerosolized route of administration takes longer to produce fibrotic lesions (40 to 120 days) (39). Interestingly, silica can induce a different fibrotic response in rats and mice. In rats the silica instillation caused chronic and progressive inflammation accompanied by overproduction of TNF- $\alpha$, which was effectively blocked by anti-inflammatory therapy. In contrast, in mice the fibrotic response was associated with a limited and transient inflammation and overexpression of the anti-inflammatory cytokine IL-10 and anti-inflammatory therapy had no effect (40).

Advantages and disadvantages of the model: The greatest advantage is the persistence of fibrotic lesions as a result of decreased clearance of the pulmonary silica particles (41). However, this model requires a costly aerosol delivery device (if aerosolized) and a longer period for fibrosis development (4-16 weeks), while the reproducibility of this model is limited because of absence of several characteristic lesions of UIP and pulmonary conditions resembling more the lung silicosis than the lung fibrosis $(1,3)$.

\section{Asbestos model}

Another model that recaptures the important form of human lung fibrosis is an exposure to asbestos (42). Asbestos-induced pulmonary fibrosis models clearly differ from IPF by multiple histological findings. In some cases the UIP pattern may also be present (4). Following intratracheal administration of asbestos fibers, fibrosis is generated more centrally than subpleurally and changes in lungs are often unevenly distributed, while inhalation models form a pattern of damage to peripheral areas, as well. However, development of the disease may be prolonged, especially when chrysotile fibres are used (43). The deposition of asbestos fibres triggers fibrotic changes by an increased apoptosis of alveolar epithelial cells and by overproduction of profibrotic cytokines by activated T-cells, all events leading to differentiation of myofibroblasts and production of extracellular matrix (44).

Advantages and disadvantages of the model: The advantage is the persistence of fibrotic lesions. However, similarly to silica model, the reproducibility of this model is limited because of pulmonary conditions resembling more the lung asbestosis than the lung fibrosis. 


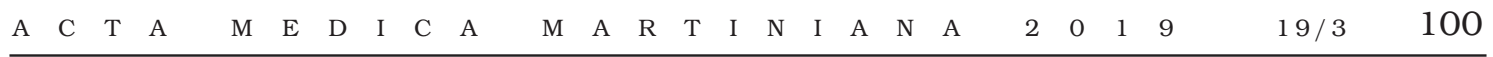

\section{CONCLUSION}

Animal models of pulmonary fibrosis are an important tool for decrypting many aspects of pulmonary fibrosis pathogenesis. They represent irreplaceable preclinical models for hypothesis testing as well as for administration of potentially beneficial treatments. However, the diversity of individual experimental models of fibrosis requires that their advantages and limitations should be carefully considered before each experiment or project.

\section{REFERENCES}

1. Moore BB, Hogaboam CM. Animal model of human lung disease. Am J Physiol Lung Cell Mol Physiol 2008; 294: 152-60.

2. Fujimoto H, Kobayashi T, Azuma A. Idiopathic Pulmonary Fibrosis: treatment and prognosis. Clin Med Insights Circ Respir Pulm Med 2016; 9: 179-85.

3. Carrington R, Jordan S, Pitchford SC, Page CP. Use of animal models in IPF research. Pulm Pharmacol Ther 2018; 51: 73-8.

4. Sanchez VC, Pietruska JR, Miselis NR, et al. Biopersistence and potential adverse health impacts of fibrous nanomaterials: what have we learned from asbestos? Wiley Interdiscip Rev Nanomed Nanobiotechnol 2009; 1: 511-29.

5. Takita T, Muraoka Y, Nakatani T, et al. Metal-complex of bleomycin and its implication for the mechanism of bleomycin action. J Antibiot 1978; 31: 1073-7.

6. Della Latta V, Cecchettini A, Del Ry S, et al. Bleomycin in the setting of lung fibrosis induction: from biological mechanisms to counteractions. Pharmacol Res 2015; 97: 122-30.

7. Claussen CA, Long EC. Nucleic Acid recognition by metal complexes of bleomycin. Chem Rev 1999; 99: 2797-816.

8. Sato K, Tashiro Y, Chibana S, et al. Role of lipid-derived free radical in bleomycin-induced lung injury in mice: availability for ESR spin trap method with organic phase extraction. Biol Pharm Bull 2008; 31: 1855-9.

9. Chaudhary NI, Schnapp A, Park JE. Pharmacologic differentiation of inflammation and fibrosis in the rat bleomycin model. Am J Respir Crit Care Med 2006; 173: 769-76.

10. Sebti SM, Mignano JE, Jani JP, et al. Bleomycin hydrolase: molecular cloning, sequencing, and biochemical studies reveal membership in the cysteine proteinase family. Biochemistry 1989; 28 : 6544-8.

11. Tomášková V Jr, Rozborilová E, Flochová E, Staško J, Kubisz P. Effect of cytostatic drugs on the endothelium and platelets. Acta Med Mart 2002; 2: 20-3.

12. Fleischman RW, Baker JR, Thompson GR, et al. Bleomycin-induced interstitial pneumonia in dogs. Thorax 1971; 26: 675-82.

13. Adamson IY, Bowden DH. The pathogenesis of bleomycin-induced pulmonary fibrosis in mice. Am J Pathol 1974; 77: 185-97.

14. Thrall RS, Mccormick JR, Jack RM, et al. Bleomycin-induced pulmonary fibrosis in the rat: inhibition by indomethacin. Am J Pathol 1979; 95: 117-30.

15. Bale S, Sunkoju M, Reddy SS, et al. Oropharyngeal aspiration of bleomycin: An alternative experimental model of pulmonary fibrosis developed in Swiss mice. Indian J Pharmacol 2016; 48: 643-8.

16. Egger C, Cannet C, Gérard C, et al. Administration of Bleomycin via the Oropharyngeal Aspiration Route Leads to Sustained Lung Fibrosis in Mice and Rats as Quantified by UTE-MRI and Histology. PLoS One 2013; 8: e63432.

17. Zhao J, Shi W, Wang YL, et al. Smad3 deficiency attenuates bleomycin-induced pulmonary fibrosis in mice. Am J Physiol Lung Cell Mol Physiol 2002; 282: 585-93.

18. Wollin L, Wex E, Pautsch A, et al. Mode of action of nintedanib in the treatment of idiopathic pulmonary fibrosis. Eur Respir J 2015; 45: 1434-45. 
19. Wollin L, Maillet I, Quesniaux V, et al. Antifibrotic and anti-inflammatory activity of the tyrosine kinase inhibitor nintedanib in experimental models of lung fibrosis. J Pharmacol Exp Ther 2014; 349: 209-20.

20. Oku H, Shimizu T, Kawabata T, et al. Antifibrotic action of pirfenidone and prednisolone: different effects on pulmonary cytokines and growth factors in bleomycin-induced murine pulmonary fibrosis. Eur J Pharmacol 2008; 590: 400-8.

21. Maher TM. Pirfenidone in idiopathic pulmonary fibrosis. Drugs Today (Barc) 2010; 46: 473-82.

22. Liu W, Wan J, Han JZ, et al. Antiflammin-1 attenuates bleomycin-induced pulmonary fibrosis in mice. Respir Res 2013; 14: 101.

23. Li YJ, Azuma A, Usuki J, et al. EM703 improves bleomycin-induced pulmonary fibrosis in mice by the inhibition of TGF-beta signaling in lung fibroblasts. Respir Res 2006; 7: 16.

24. Ji Y, Dou YN, Zhao QW, et al. Paeoniflorin suppresses TGF- mediated epithelial-mesenchymal transition in pulmonary fibrosis through a Smad-dependent pathway. Acta Pharmacol Sin 2016; 37: 794-804.

25. Tang $\mathrm{H}, \mathrm{He} \mathrm{H}$, Ji H, et al. Tanshinone IIA ameliorates bleomycin-induced pulmonary fibrosis and inhibits transforming growth factor-beta- -dependent epithelial to mesenchymal transition. J Surg Res 2015; 197: 167-75.

26. Degryse AL, Tanjore $\mathrm{H}, \mathrm{Xu} \mathrm{XC}$, et al. Repetitive intratracheal bleomycin models several features of idiopathic pulmonary fibrosis. Am J Physiol Lung Cell Mol Physiol 2010; 299: 442-52.

27. Izbicki G, Segel MJ, Christensen TG, et al. Time course of bleomycin-induced lung fibrosis. Int J Exp Pathol 2002; 83: 111-9.

28. Peng R, Sridhar S, Tyagi G, et al. Bleomycin induces molecular changes directly relevant to idiopathic pulmonary fibrosis: a model for "active" disease. PLoS One 2013; 8: e59348.

29. Chua F, Gauldie J, Laurent GJ. Pulmonary fibrosis: searching for model answers. Am J Respir Cell Mol Biol 2005; 33: 9-13.

30. Moore BB, Murray L, Das A, et al. The role of CCL12 in the recruitment of fibrocytes and lung fibrosis. Am J Respir Cell Mol Biol 2006; 35: 175-81.

31. Roberts SN, Howie SE, Wallace WA, et al. A novel model for human interstitial lung disease: hapten-driven lung fibrosis in rodents. J Pathol 1995; 176: 309-18.

32. Christensen PJ, Goodman RE, Pastoriza L, et al. Induction of lung fibrosis in the mouse by intratracheal instillation of fluorescein isothiocyanate is not T-cell-dependent. Am J Pathol 1999; 155: 1773-9.

33. Degryse AL, Lawson WE. Progress toward improving animal models for idiopathic pulmonary fibrosis. Am J Med Sci 2011; 341: 444-9.

34. Karvonen RL, Fernandez-Madrid F, Maughan RL, et al. An animal model of pulmonary radiation fibrosis with biochemical, physiologic, immunologic, and morphologic observations. Radiat Res 1987; 111: 68-80.

35. Haston CK, Travis EL. Murine susceptibility to radiation-induced pulmonary fibrosis is influenced by a genetic factor implicated in susceptibility to bleomycin-induced pulmonary fibrosis. Cancer Res 1997; 57: 5286-91.

36. Morgan GW, Breit SN. Radiation and the lung: a reevaluation of the mechanisms mediating pulmonary injury. Int J Radiat Oncol Biol Phys 1995; 31: 361-9.

37. Paun A, Kunwar A, Haston CK. Acute adaptive immune response correlates with late radiationinduced pulmonary fibrosis in mice. Radiat Oncol 2015; 10: 45.

38. Lakatos HF, Burgess HA, Thatcher TH, et al. Oropharyngeal aspiration of a silica suspension produces a superior model of silicosis in the mouse when compared to intratracheal instillation. Exp Lung Res 2006; 32: 181-99.

39. Davis GS, Leslie KO, Hemenway DR. Silicosis in mice: effects of dose, time, and genetic strain. J Environ Pathol Toxicol Oncol 1998; 17: 81-97.

40. Barbarin V, Nihoul A, Misson P, et al. The role of pro- and anti-inflammatory responses in silica-induced lung fibrosis. Respir Res 2005; 6: 112.

41. Barbarin V, Arras M, Misson P, et al. Characterization of the effect of interleukin-10 on silica-induced lung fibrosis in mice. Am J Respir Cell Mol Biol 2004; 31: 78-85. 


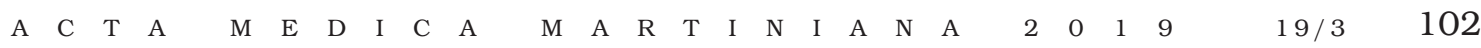

42. Hurbánková M, Kaiglová A, Buchancová J. Cytokines - the important biomarkers of lung injury after exposure to indstrial fibrous dusts. Acta Med Mart 2001; 1: 19-24.

43. Roggli V, Gibbs AR, Attanoos R, et al. Pathology of asbestosis: an update of the diagnostic criteria response to a critique. Arch Pathol Lab Med 2016; 140: 950-2.

44. Kim SJ, Cheresh P, Jablonski RP, et al. The role of mitochondrial DNA in mediating alveolar epithelial cell apoptosis and pulmonary fibrosis. Int J Mol Sci 2015; 16: 21486-519.

\section{Acknowledgments}

The work was supported by projects VEGA 1/0356/18 and APVV-15-0075.

Received: September, 28, 2019

Accepted: November, 6, 2019 